\title{
Non-Markovian super-superradiance in a linear chain of up to 100 qubits
}

\author{
Fatih Dinc $\odot^{*}$ and Agata M. Brańczyk $\odot$ \\ Perimeter Institute for Theoretical Physics, Waterloo, Ontario, Canada N2L $2 Y 5$
}

(Received 13 September 2019; published 19 December 2019)

\begin{abstract}
We study non-Markovian enhancement effects in the spontaneous emission of a collective excitation in a linear chain of up to 100 qubits coupled to a one-dimensional waveguide. We find that for a critical separation of qubits, the system exhibits super-superradiant (SSR) behavior leading to collective decay stronger than the usual Dicke superradiance. Here time-delayed coherent quantum feedback effects are at play on top of the usual Dicke superradiance effects. We find a linear scaling for the SSR decay rate with increasing qubit number $N$ such that $\Gamma_{\mathrm{SSR}} \sim 2.277 N \gamma_{0}$, where $\gamma_{0}$ is the single-emitter decay rate to a one-dimensional waveguide, as opposed to $\Gamma_{\text {Dicke }} \sim N \gamma_{0}$ for Dicke superradiance. The SSR decay rate can be tuned with qubit separation distance and may therefore have application for quantum technologies.
\end{abstract}

DOI: 10.1103/PhysRevResearch.1.032042

Surprising phenomena can emerge when multiple quantum systems coordinate their behavior. A well-known example of this, Dicke superradiance [1,2], occurs when a group of $N$ quantum emitters, excited in a symmetric state, exhibits enhanced spontaneous emission [3-5]. This phenomenon, predicted when the distance between emitters is small compared to the wavelength of radiation, can be understood as collective behavior of the entire system. Curiously, enhanced emission can also occur when the emitters are macroscopically separated [6-9], a regime in which collective behavior might not be expected. Furthermore, the emission can become more enhanced than Dicke superradiance, earning it the name super-superradiance (SSR) [8]. This highly counterintuitive phenomenon can be explained by an effect that has recently gained attention in other contexts [10-16]: time-delayed coherent quantum feedback. In this paper, we study the effects of time-delayed coherent quantum feedback on the supersuperradiant collective decay rate of a linear chain of qubits coupled to a one-dimensional (1D) waveguide.

The 1D geometry of the waveguide enhances scattering and is thus particularly favorable to study time-delayed coherent quantum feedback. While linear chains of qubits in 1D waveguides have been studied extensively, both theoretically $[9,17-$ 21] and experimentally [22-24], the literature mostly focuses on regimes where the Markovian approximation is valid, i.e., time-retardation effects are negligible. Time-delayed coherent quantum feedback, however, occurs when qubits are separated by distances in which the Markovian limit is no longer valid. One must therefore take into account non-Markovian dynamics of qubit-light interactions. This was done in waveguide

\footnotetext{
*Present address: Department of Applied Physics, Stanford University, Stanford, CA, USA; fdinc@ @stanford.edu

Published by the American Physical Society under the terms of the Creative Commons Attribution 4.0 International license. Further distribution of this work must maintain attribution to the author(s) and the published article's title, journal citation, and DOI.
}

QED for a single qubit in [13,25-27], for two qubits in [6,7], and for three qubits in [8]. Here we consider up to 100 qubits.

We consider a linear chain of $N$ identical qubits separated by a distance $L$ and coupled to a $1 \mathrm{D}$ waveguide as shown in Fig. 1. We study SSR by computing the collective decay rates of the $N$-qubit system. Our method of choice is the real-space approach [8]. In this approach, one first identifies the transfer matrix for the system and then uses that matrix to write down and solve a characteristic equation for the collective decay rates. The real-space approach has the following strengths for this problem: (i) no need to model time dynamics, as the collective decay rates can be found from steady-state single-frequency solutions (energy eigenstates for the whole system); (ii) no need to identify the multiqubit superposition states that correspond to SSR (in fact, one does not need to consider the qubit excitation subspace at all); and (iii) energy eigenstates can be found via a recursive transfer matrix method. These strengths of the real-space approach make the problem significantly more tractable for large- $N$ systems.

We start by identifying the transfer matrix. For a plane wave with frequency $k$, incident from the far left, the transformation that relates the transmitted and reflected photon coefficients $t$ and $r$, respectively, to the incident photon is

$$
\left(\begin{array}{l}
1 \\
r
\end{array}\right)=T^{N}\left(\begin{array}{l}
t \\
0
\end{array}\right)
$$

Here $T$ is the transfer matrix corresponding to a unit cell, which consists of interqubit propagation and a single qubitlight interaction. Following [8], $T$ can be written as

$$
T=\left(\begin{array}{cc}
1+i \frac{\gamma_{0}}{2 \Delta_{k}} & i \frac{\gamma_{0}}{2 \Delta_{k}} \\
-i \frac{\gamma_{0}}{2 \Delta_{k}} & 1-i \frac{\gamma_{0}}{2 \Delta_{k}}
\end{array}\right)\left(\begin{array}{cc}
e^{-i k L} & 0 \\
0 & e^{i k L}
\end{array}\right),
$$

where $L$ is the distance between two adjacent qubits, $\Delta_{k}=$ $E_{k}-\Omega$ is the detuning between the photon energy $E_{k}$ and the qubit energy separation $\Omega$, and $\gamma_{0}$ is the decay rate of a single qubit to the $1 \mathrm{D}$ waveguide. When deriving this transfer matrix, we use the real-space Hamiltonian [8,20,28], where the lightmatter interactions take the form of a $\delta$ function at the qubit 


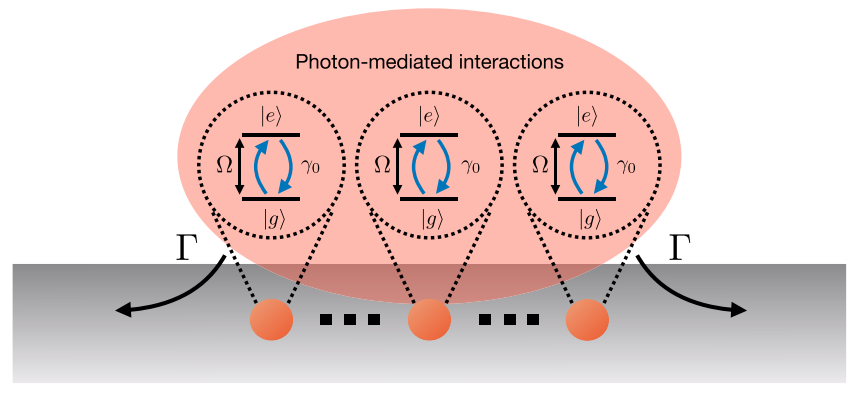

FIG. 1. Linear chain of $N$ qubits coupled to a 1D waveguide. The photon-mediated interactions between qubits lead to a collective behavior of the system described by collective decay rates. An initially excited system decays through these decay modes.

position. For the scope of this paper, we assume $\gamma_{0} / \Omega \ll 1$, in alignment with the rotating-wave approximation performed at the Hamiltonian level. For the remainder of this paper, we set $\hbar=1$ and $v_{g}=1$, where $v_{g}$ is the group velocity of the photons inside the waveguide.

We now determine the characteristic equation for the collective decay rates by setting the first element of the transfer matrix in (1) to zero: $\left(T^{N}\right)_{11}=0$. The decay rates $\Gamma$ can then be obtained via a complex rotation followed by a scaling from the poles $\Delta_{k}^{(p)}$ of the scattering parameters, i.e., $\Gamma=2 i \Delta_{k}^{(p)}$ [8] (the real part of $\Gamma$ corresponds to the physical decay rate and the imaginary part is responsible for a characteristic frequency shift). The factor of 2 comes from the fact that we are interested in population decay rates. With this definition, the characteristic equation of the poles for an $N$-qubit system is

$$
f\left(\Delta_{k}\right)=\Delta_{k}^{N}\left(T^{N}\right)_{11}
$$

where the multiplication with $\Delta_{k}^{N}$ results in a characteristic equation that is polynomial in $\Delta_{k}$ and $e^{i k L}$ (hence analytic in the complex plane). As a result, the problem of finding the collective decay rates comes down to finding the roots of the function $f\left(\Delta_{k}\right)$. Solving this characteristic equation for a single qubit and performing the rotation, we find the single-emitter decay rate to be $\gamma_{0}$, as expected.

One can consider this problem of finding the collective decay rates in two different regimes: the Markovian regime in which the qubits are microscopically separated $\left[L \sim O\left(\Omega^{-1}\right)\right]$ and the propagation time of the photons within the system is negligible and the non-Markovian regime in which the qubits are macroscopically separated $\left[L \sim O\left(\gamma_{0}^{-1}\right)\right]$ and the propagation time of the photons within the system is not negligible. The superradiance condition in both regimes is $\Omega L=\pi n$, where $n$ is an integer [8,9].

In the Markovian regime, the propagation phase acquired by the photon between two qubits can be linearized, $k L \simeq \Omega L$ $[6,8]$. In this regime, $f\left(\Delta_{k}\right)$ can be approximated by an $N$ th degree polynomial in $\Delta_{k}$, since the exponential terms in the transfer matrix can be replaced by a constant $e^{i k L} \simeq e^{i \Omega L}$. The $N$ zeros, which correspond to $N$ collective decay rates, can be found analytically. When the superradiant condition is satisfied, the superradiant decay rate is $N \gamma_{0}$ and all other decay rates are zero [8]. This is the case of Dicke superradiance.
In the non-Markovian regime, the photon's propagation phase can no longer be linearized, so the Markovian approximation is no longer valid. In this regime, $f\left(\Delta_{k}\right)$ can no longer be approximated by a polynomial (it depends on $e^{i k L}=$ $e^{i \Omega L\left(1+\Delta_{k} / \Omega\right)}$ as well). Relaxing the linearization condition leads to time-retardation effects inside the multiqubit system. A discussion on how the linearization $k L \simeq \Omega L$ is linked to the Markovianity assumption can be found in [8] (in particular, in Secs. VI and VIII). Consequently, $f\left(\Delta_{k}\right)$ has infinitely many zeros that correspond to infinitely many collective decay rates, which can be divided into two categories: those that tend to Markovian decay rates in the limit $L \rightarrow 0$ (we call these Markovian-like) and those that tend to infinity in the same limit (we call these exclusively non-Markovian).

Super-superradiance refers to the phenomenon that a Markovian-like decay rate surpasses the Dicke superradiance decay rate $\Gamma_{\text {Dicke }}=N \gamma_{0}$. To achieve maximum SSR, the qubits must be separated by a critical distance $L_{c}$ (as pointed out in [7]) as well as satisfy the condition $\Omega L_{c}=n \pi$. For any $L$ and $\Omega L=n \pi, f\left(\Delta_{k}\right)$ has $N-1$ zeros that correspond to $\Gamma=0$ (this occurs when $\Delta_{k}=0$ ). This leaves only one Markovian-like decay rate which could potentially become SSR, i.e., the nonzero $\Gamma$ closest to the origin.

In our analysis, we therefore first assume that $\Omega L=n \pi$ and set $e^{i k L}=e^{i \Omega L\left(1+\Delta_{k} / \Omega\right)}=(-1)^{n} e^{i \Delta_{k} L}$. We then use a numerical root finding algorithm to find the first $N$ zeros of $f\left(\Delta_{k}\right)$ that are closest to the origin; $N-1$ of such zeros are found to be within the vicinity of $\Delta_{k}=0$, deviating only by small amounts due to numerical imprecision. The $N$ th zero, labeled as $p$, gives the superradiant decay rate via the Wick-like rotation $\Gamma_{u}=2 i p$. The real part of $\Gamma_{u}$ gives the physical decay rate. We define the SSR decay rate as $\Gamma_{\mathrm{SSR}}=$ $\max _{L} \operatorname{Re}\left[\Gamma_{u}\right]$ and perform the maximization numerically. As a sanity check, we then plot $\ln \left[\left|f\left(\Delta_{k}\right)\right|\right]$ for $\Delta_{k}$ near $\Gamma_{\mathrm{SSR}}$ and compare it with the values found numerically.

Figure 2 shows the symmetric and antisymmetric decay rates, i.e., those that couple to symmetric and antisymmetric superposition states, for $N=2$ qubits in the non-Markovian regime as a function of qubit separation $L$ for finite $\Omega$, enveloped by an upper bound given by $\Omega \rightarrow \infty$ (for $L=0$, the symmetric decay rate couples to the Dicke state). The symmetric and antisymmetric decay rates follow the same trend as in Fig. 4(d) of [6]. The numerical values $\Gamma_{\text {SSR }} \approx$ $4.59 \gamma_{0}$ and $L_{c} \approx 0.56 \gamma_{0}^{-1}$ match perfectly with the findings of [7]. When the condition $\Omega L_{c}=n \pi$ is satisfied, the peak of the curve for the symmetric decay rate lines up with the peak of the dashed blue envelope function, as is the case in Fig. 2. If the condition is not satisfied, the system acquires a maximum decay for a distance closest to $L_{c}$.

We now ask whether SSR is a general phenomenon that persists beyond the known cases of $N=2$ and $N=3$. To answer this, we repeat the above analysis for various $N$ up to $N=100$. We find that indeed SSR persists for large $N$ and that $\Gamma_{\text {SSR }}$ scales linearly, as shown in Fig. 3(a). The scaling is similar to that of Dicke superradiance. Figure 3(b) shows how the critical distance $L_{c}$ scales with $N$. The fit is almost linear, but deviates for $N<10$.

To study the closeness of the linear fits, we plot the deviation of the numerical results in Fig. 3. For $\Gamma_{\mathrm{SSR}}$, the deviation hovers around $\sim 10^{-6}$ for large $N$, which is within 


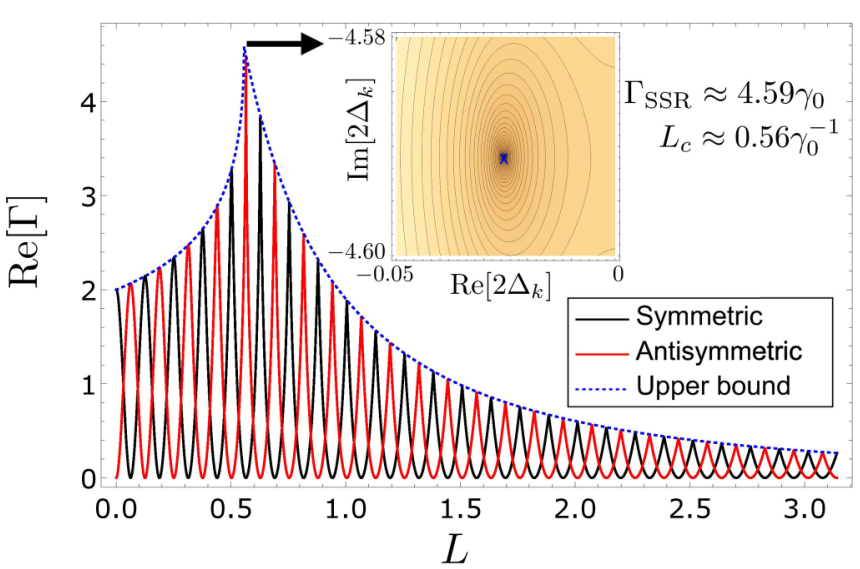

FIG. 2. Physical decay rate, given by the real part of $\Gamma$ (in units of $\gamma_{0}$ ) for $N=2$ qubits in the non-Markovian regime as a function of qubit separation $L$ (in units of $\gamma_{0}^{-1}$ ). Black lines $\left(\operatorname{Re}[\Gamma]=2 \gamma_{0}\right.$ at $L=$ 0 ) and red lines $(\operatorname{Re}[\Gamma]=0$ at $L=0)$ correspond, respectively, to symmetric and antisymmetric decay rates for $\Omega=50 \gamma_{0}$. The dashed blue line gives the upper bound obtained in the limit $\Omega \rightarrow \infty$ of the superradiant decay values. All numerical values are normalized with respect to $\gamma_{0}$. The inset shows $\ln \left[\left|f\left(\Delta_{k}\right)\right|\right]$ for $L=L_{c}$ for $\Delta_{k}$ close to the SSR pole.

the numerical precision of our algorithm. For $L_{c}$, the fit is not as close: The $N^{-2}$ scaling fits the data within $0.1 \%$. For more evidence for this scaling, we turn to analytical investigations.

A closed-form solution exists for the transmission and reflection coefficients related by the transfer matrix as in (1) [20]. Applying the superradiant condition $\Omega L=\pi n$, the characteristic set of equations corresponding to the poles becomes

$$
\begin{aligned}
\cos (\lambda) & =\cos (p L)+\frac{\gamma_{0}}{2 p} \sin (p L), \\
\left(p+i \gamma_{0} / 2\right) \sin (\lambda N) & =e^{i p L} p \sin [\lambda(N-1)] .
\end{aligned}
$$

Here $\lambda$ is a complex number that relates both equations and $p$ relates to the collective decay rate as $\Gamma=2 i p$. Now let us set $p=-i \frac{\alpha}{2} N \gamma_{0}\left(\Gamma=\alpha N \gamma_{0}\right)$ and $L=\beta N^{-2} \gamma_{0}^{-1}$ from the numerical fit we obtained in Fig. 3. Here $\alpha$ and $\beta$ are free parameters to be determined. Then, neglecting terms of $O\left(N^{-1}\right)$, the set of equations (4) becomes

$$
g(\alpha, \beta)=2 \alpha \tau \cosh (\tau)-\left(2+\alpha^{2} \beta\right) \sinh (\tau)=0,
$$

with $\tau=0.5 \sqrt{\beta\left(4+\alpha^{2} \beta\right)}$. We note that the coefficient of $O\left(N^{-1}\right)$ term is nonzero, which explains the deviations for $N<10$. The SSR decay rate scales asymptotically for large $N$ in contrast to the exact scaling of the Dicke superradiance. There are infinitely many $(\alpha, \beta)$ pairs that satisfy this equation, which has been illustrated in Fig. 4. The most important observation is that there exists a $\beta_{c}$ such that there is no solution $^{1}$ to $g(\alpha, \beta)=0$ for $\beta>\beta_{c}$ and there are two pairs $\left(\alpha_{s / l}, \beta\right)\left(s / l\right.$ stand for small/large) for $\beta<\beta_{c}$. In this case, the Markovian-like decay rate scales as $\Gamma_{u}=\alpha_{s} N \gamma_{0}$ and the

\footnotetext{
${ }^{1}$ This has been checked numerically for a large interval of $\alpha$ and $\beta$ values.
}
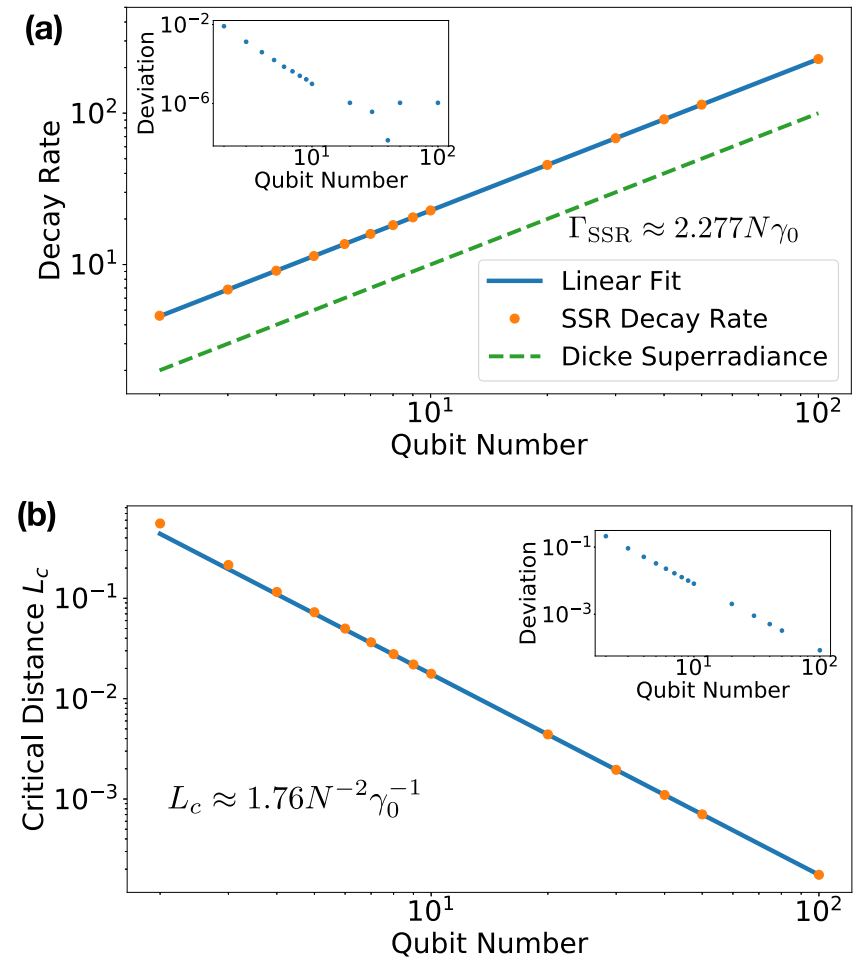

FIG. 3. Scaling of (a) the SSR decay rate $\Gamma_{\text {SSR }}$ and (b) the critical distance $L_{c}$ with respect to the qubit number $N$. The insets show the relative deviation between the fit and the numerical values $\left(\left|\frac{\text { fit-data }}{\text { data }}\right|\right)$. The uncertainty in the scaling of the SSR decay rate is well within the errors of the numerical maximization algorithm, whereas for the critical distance there is a significant deviation from the fit even for large $N$. We note that, unlike Dicke superradiance, the SSR does not scale exactly linearly for small $N$. There is a significant difference for $N \leqslant 10$ between the fit and the numerical data.

$(N+1)$ th (exclusively non-Markovian) decay rate scales as $\Gamma_{\mathrm{NM}}=\alpha_{l} N \gamma_{0}$. As $\beta \rightarrow \beta_{c}$, both $\Gamma_{u}$ and $\Gamma_{\mathrm{NM}}$ come closer and right at $\beta=\beta_{c}, \Gamma_{u}$ is maximized and has the same real part as $\Gamma_{\mathrm{NM}}$. Consequently, $\Gamma_{\mathrm{SSR}}=\max _{L} \operatorname{Re}\left[\Gamma_{u}\right]$ is obtained at the position $\frac{\partial g(\alpha, \beta)}{\partial \alpha}=0$. This corresponds to the condition $\alpha_{c} \beta_{c}=$ 4 , with $c$ standing for the critical values. The characteristic equation can be solved for this condition and the resulting $\left(\alpha_{c}, \beta_{c}\right) \approx(2.277,1.76)$ pair agrees with the numerical fit in Fig. 3, confirming our numerical analysis. It is important to note that the crucial step here is the ansatz that $p \sim N \gamma_{0}$ and $L \sim N^{-2} \gamma_{0}^{-1}$, which was obtained from the numerical analysis in the first place.

This analytical investigation also explains another interesting phenomenon. The imaginary part of $\Gamma_{\mathrm{SSR}}$ is responsible for the collective energy level shifts. For $L \ll L_{c}$, the superradiant decay rate $\Gamma_{u}$ is strictly real and does not have an imaginary component, whereas the $(N+1)$ th exclusively non-Markovian collective decay rate is negligibly far away from the origin. This justifies the $N$-pole approximation performed in [6], where the $(N+1)$ th pole is so far away from the first $N$ such that it can be neglected. However, for $L>L_{c}$, the $N$ th and $(N+1)$ th decay rates become degenerate. They have the same real parts, but also imaginary parts with the opposite sign. The fact that the decay rates acquire imaginary components for $L>L_{c}$ suggests a shift in collective energy 


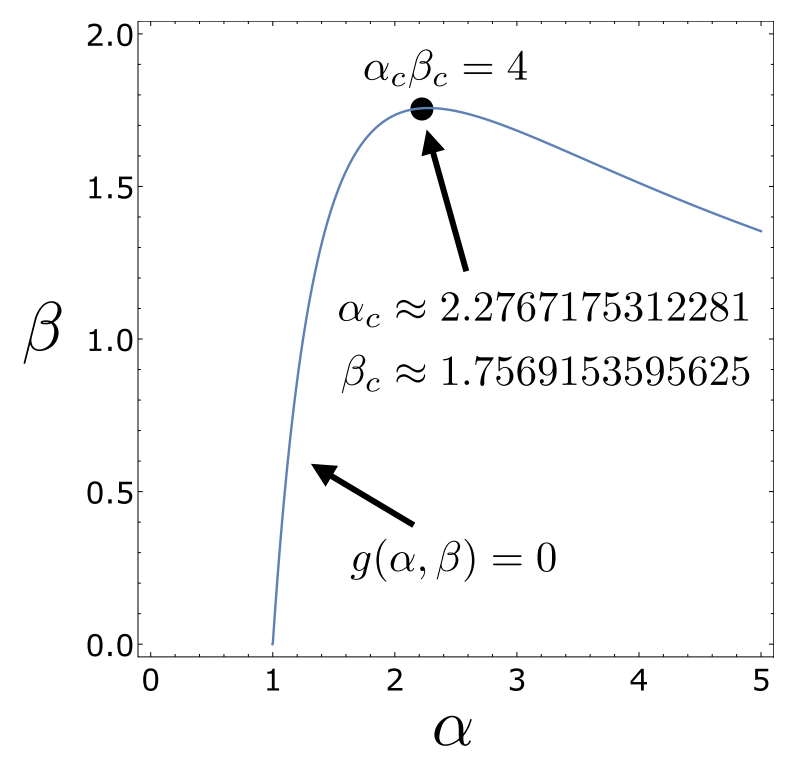

FIG. 4. Contour plot of $g(\alpha, \beta)=0$. Here the assumption $\Delta_{k}=$ $-i 0.5 \alpha N \gamma_{0}$ and $L_{c}=\beta N^{-2} \gamma_{0}^{-1}$ is valid as long as we pick a pair $(\alpha, \beta)$ on the contour. For $\beta>\beta_{c}$, there is no such pair. For $\beta<\beta_{c}$, there are two such pairs corresponding to two distinct poles (one exclusively non-Markovian, one Markovian-like). The pole with smaller $\alpha$ corresponds to $\Gamma_{u}$, but is not the SSR decay rate since larger $\alpha$ can be achieved for $\Gamma_{u}=\alpha N \gamma_{0}$ (and $\Gamma_{\mathrm{SSR}}=\max _{\beta} \operatorname{Re}\left[\Gamma_{u}\right]$ ). For $\beta=\beta_{c}$, there is only one such pair, corresponding to $\Gamma_{\mathrm{SSR}}$ and $L_{c}$. Note that for the pair $\left(\beta_{c}, \alpha_{c}\right)$, the equation $\alpha_{c} \beta_{c}=4$ is an exact one.

levels analogous to the Lamb shift. A similar observation has been made in [6] for $N=2$ qubits. Furthermore, we point to another interesting phenomenon. The energy levels corresponding to the $N$ th closest pole to the origin changes discontinuously around the superradiant condition $\Omega L=n \pi$ with changing $L$ for $L>L_{c}$. This has been plotted in Fig. 4 of [6] for $N=2$, where increasing the distance between qubits can lead to discontinuous changes in the energy level shifts around $L$ values where $\Omega L=n \pi$. We observe here that this is a more general phenomenon for any $N$. An investigation of the shifts in the collective energy levels due to non-Markovian effects is left for future work.

In summary, the SSR decay rate scales linearly $\left(\Gamma_{\mathrm{SSR}} \sim\right.$ $N \gamma_{0}$ ) and the critical qubit separation scales by the inverse square $\left(L_{c} \sim N^{-2} \gamma_{0}^{-1}\right)$. While the time-delayed quantum coherent feedback leads to a stronger decay of the collective system, the scaling of the decay rate is still linear with increasing $N$. Furthermore, while $\Gamma_{\text {Dicke }} \sim N \gamma_{0}$ fits exactly for any $N$ in the case of the Dicke superradiance, the value $\Gamma_{\text {SSR }} \sim 2.277 N \gamma_{0}$ fits well asymptotically for large $N$. We do not yet know the origin of the linear scaling, but it would be interesting to investigate this interesting phenomenon further. As a starting point, it is important to realize that the overall size of the system is approximately equal to $N L_{c} \sim$ $1.76 N^{-1} \gamma_{0}^{-1} \ll \gamma_{0}^{-1}$. This means that light initially emitted by a qubit at one end of the chain travels all the way to the other end and back in less time than the half-time of that individual qubit. Consequently, even photon-mediated interactions between the first and $N$ th qubits are in fact significant in the emergence of the SSR phenomenon. Conversely, when qubits are separated by large distances, the collective decay rates become subradiant, in agreement with the findings of [6]. Hence, SSR is only observed when the qubits are moderately separated $\left(L \approx L_{c}\right)$, where both collective interactions and time-retardation effects are at play.

When it comes to applications, the non-Markovian system provides additional control parameters. In Markovian 1D waveguide QED, the decay rates depend on the coupling and the phase parameter $\Omega L$. In the non-Markovian regime, however, the decay rates also depend on the qubit separation $L$ and frequency $\Omega$ independently. Furthermore, the distance can enhance the decay rate beyond Dicke superradiance. By tuning the qubit separation, one can tune the interaction between photons and multiqubit systems, which might have application for quantum technologies such as quantum memories [29-31], quantum gates [32], and pulse shaping [8]. Furthermore, our formalism makes it possible to study systems with many qubits. We thus expect our approach to be useful for designing new quantum technologies.

Research at the Perimeter Institute was supported by the Government of Canada through the Department of Innovation, Science and Economic Development Canada and by the Province of Ontario through the Ministry of Research and Innovation. We acknowledge support from the Natural Sciences and Engineering Research Council of Canada (Grant No. RGPIN-2016-04135).
[1] A. V. Andreev, V. I. Emelyanov, and Y. A. Ilinskiĭ, Collective spontaneous emission (Dicke superradiance), Sov. Phys. Usp. 23, 493 (1980).

[2] R. H. Dicke, Coherence in spontaneous radiation processes, Phys. Rev. 93, 99 (1954).

[3] R. Fleury and A. Alu, Enhanced superradiance in epsilon-nearzero plasmonic channels, Phys. Rev. B 87, 201101 (2013).

[4] S. John and T. Quang, Localization of Superradiance Near a Photonic Band Gap, Phys. Rev. Lett. 74, 3419 (1995).
[5] N. Vats and S. John, Non-Markovian quantum fluctuations and superradiance near a photonic band edge, Phys. Rev. A 58, 4168 (1998).

[6] H. Zheng and H. U. Baranger, Persistent Quantum Beats and Long-Distance Entanglement from Waveguide-Mediated Interactions, Phys. Rev. Lett. 110, 113601 (2013).

[7] K. Sinha, P. Meystre, E. A. Goldschmidt, F. K. Fatemi, S. L. Rolston, and P. Solano, Non-Markovian collective emission from macroscopically separated emitters, arXiv:1907.12017. 
[8] F. Dinc, İ. Ercan, and A. M. Brańczyk, Exact Markovian and non-Markovian time dynamics in waveguide QED: Collective interactions, bound states in continuum, superradiance and subradiance, Quantum 3, 213 (2019).

[9] Y. Zhou, Z. Chen, and J.-T. Shen, Single-photon superradiant emission rate scaling for atoms trapped in a photonic waveguide, Phys. Rev. A 95, 043832 (2017).

[10] A. L. Grimsmo, Time-Delayed Quantum Feedback Control, Phys. Rev. Lett. 115, 060402 (2015).

[11] P.-O. Guimond, M. Pletyukhov, H. Pichler, and P. Zoller, Delayed coherent quantum feedback from a scattering theory and a matrix product state perspective, Quantum Sci. Technol. 2, 044012 (2017).

[12] H. Pichler and P. Zoller, Photonic Circuits with Time Delays and Quantum Feedback, Phys. Rev. Lett. 116, 093601 (2016).

[13] G. Calajó, Y.-L. L. Fang, H. U. Baranger, F. Ciccarello et al., Exciting a Bound State in the Continuum Through Multiphoton Scattering Plus Delayed Quantum Feedback, Phys. Rev. Lett. 122, 073601 (2019).

[14] H. Pichler, S. Choi, P. Zoller, and M. D. Lukin, Universal photonic quantum computation via time-delayed feedback, Proc. Natl. Acad. Sci. USA 114, 11362 (2017).

[15] S. J. Whalen, A. L. Grimsmo, and H. J. Carmichael, Open quantum systems with delayed coherent feedback, Quantum Sci. Technol. 2, 044008 (2017).

[16] H. Chalabi and E. Waks, Interaction of photons with a coupled atom-cavity system through a bidirectional time-delayed feedback, Phys. Rev. A 98, 063832 (2018).

[17] G.-Z. Song, E. Munro, W. Nie, L.-C. Kwek, F.-G. Deng, and G.-L. Long, Photon transport mediated by an atomic chain trapped along a photonic crystal waveguide, Phys. Rev. A 98, 023814 (2018).

[18] S. Das, V. E. Elfving, F. Reiter, and A. S. Sørensen, Photon scattering from a system of multilevel quantum emitters. II. Application to emitters coupled to a one-dimensional waveguide, Phys. Rev. A 97, 043838 (2018).

[19] Z. Liao, X. Zeng, S.-Y. Zhu, and M. S. Zubairy, Single-photon transport through an atomic chain coupled to a one-dimensional nanophotonic waveguide, Phys. Rev. A 92, 023806 (2015).

[20] T. S. Tsoi and C. K. Law, Quantum interference effects of a single photon interacting with an atomic chain inside a one-dimensional waveguide, Phys. Rev. A 78, 063832 (2008).

[21] A. Albrecht, L. Henriet, A. Asenjo-Garcia, P. B. Dieterle, O. Painter, and D. E. Chang, Subradiant states of quantum bits coupled to a one-dimensional waveguide, New J. Phys. 21, 025003 (2019).

[22] A. Goban, C.-L. Hung, J. D. Hood, S.-P. Yu, J. A. Muniz, O. Painter, and H. J. Kimble, Superradiance for Atoms Trapped Along a Photonic Crystal Waveguide, Phys. Rev. Lett. 115, 063601 (2015).

[23] J. D. Hood, A. Goban, A. Asenjo-Garcia, M. Lu, S.-P. Yu, D. E. Chang, and H. J. Kimble, Atom-atom interactions around the band edge of a photonic crystal waveguide, Proc. Natl. Acad. Sci. USA 113, 10507 (2016).

[24] A. Goban, C.-L. Hung, S.-P. Yu, J. D. Hood, J. A. Muniz, J. H. Lee, M. J. Martin, A. C. McClung, K. S. Choi, D. E. Chang et al., Atom-light interactions in photonic crystals, Nat. Commun. 5, 3808 (2014)

[25] T. Tufarelli, M. S. Kim, and F. Ciccarello, Non-Markovianity of a quantum emitter in front of a mirror, Phys. Rev. A 90, 012113 (2014).

[26] T. Ramos, B. Vermersch, P. Hauke, H. Pichler, and P. Zoller, Non-Markovian dynamics in chiral quantum networks with spins and photons, Phys. Rev. A 93, 062104 (2016).

[27] Y.-L. L Fang, F. Ciccarello, and H. U Baranger, Non-Markovian dynamics of a qubit due to single-photon scattering in a waveguide, New J. Phys. 20, 043035 (2018).

[28] J.-T. Shen and S. Fan, Coherent Single Photon Transport in a One-Dimensional Waveguide Coupled with Superconducting Quantum Bits, Phys. Rev. Lett. 95, 213001 (2005).

[29] H. J. Kimble, The quantum internet, Nature (London) 453, 1023 (2008).

[30] A. I. Lvovsky, B. C. Sanders, and W. Tittel, Optical quantum memory, Nat. Photon. 3, 706 (2009).

[31] E. Saglamyurek, T. Hrushevskyi, A. Rastogi, K. Heshami, and L. J. LeBlanc, Coherent storage and manipulation of broadband photons via dynamically controlled Autler-Townes splitting, Nat. Photon. 12, 774 (2018).

[32] J. Combes and D. J. Brod, Two-photon self-Kerr nonlinearities for quantum computing and quantum optics, Phys. Rev. A 98, 062313 (2018). 